\title{
Phenomenological and microscopic cluster models II. Phase transitions
}

\author{
P. R. Fraser ${ }^{1}$, H. Yépez-Martínez ${ }^{2}$, P. O. Hess ${ }^{1}$ and G. Lévai ${ }^{3}$ \\ 1 Instituto de Ciencias Nucleares, UNAM, Circuito Exterior, C.U., \\ A.P. 70-543, 04510 México, D.F., Mexico \\ ${ }^{2}$ Universidad Autónoma de la Ciudad de México, Prolongación San Isidro 151, \\ Col. San Lorenzo Tezonco, Del. Iztapalapa, 09790 México D.F., Mexico \\ ${ }^{3}$ Institute of Nuclear Research of the Hungarian Academy of Sciences, \\ Debrecen, Pf. 51, Hungary-4001
}

\begin{abstract}
Based on the results of a previous paper (Paper I), by performing the geometrical mapping via coherent states, phase transitions are investigated and compared within two algebraic cluster models. The difference between the Semimicroscopic Algebraic Cluster Model (SACM) and the Phenomenological Algebraic Cluster Model (PACM) is that the former strictly observes the Pauli exclusion principle between the nucleons of the individual clusters, while the latter ignores it. From the technical point of view the SACM is more involved mathematically, while the formalism of the PACM is closer to that of other algebraic models with different physical content. First- and second-order phase transitions are identified in both models, while in the SACM a critical line also appears. Analytical results are complemented with numerical studies on $\alpha$-cluster states of the ${ }^{20} \mathrm{Ne}$ and ${ }^{24} \mathrm{Mg}$ nuclei.

PACS numbers: 21.60.-n,21.60.Fw,21.60.Gx
\end{abstract}

\section{INTRODUCTION}

In a former contribution [1], called from now on $\mathrm{Pa}$ per I, we investigated the geometric mapping of the Phenomenological Algebraic Cluster Model (PACM) and the Semimicroscopic Algebraic Cluster Model (SACM) 2, 3]. The first does not observe the Pauli exclusion principle while the second one does. The PACM belongs to the same family as models like the vibron model [4]. In both type of models we considered the same Hamiltonian, while the model space of each is quite different. In the SACM, observation of the Wildermuth condition [5] means that the number of relative oscillation quanta is restricted from below while in the PACM the relative oscillation quanta start from zero.

The method of geometrical mapping is gleaned from 6] for the SACM, which reduces to the usual one $7-9$ when no Pauli exclusion principle is taken into account. We showed in [1] that the differences in the mapped geometrical potential are large. However, within the PACM one can reproduce the geometric potential of the SACM by including very complicated higher-order interactions.

We also included in [1] a discussion on how to define correctly the coherent state parameter, such that for a large total number of bosons $(N$, which plays the role of a cutoff) the mapped potential will be independent of the cutoff $N$.

In this paper we concentrate on the study of phase transitions and show that not only second-order phase transitions may occur, but also first-order phase transitions. Furthermore, in the SACM a critical line appears beyond which no phase transition occurs. Thus the structure of the phase diagram of the SACM will be much richer.

We will also apply the mapping to two kind of systems, one with two spherical clusters and the other one with a deformed and a spherical cluster. Numerical studies are added. We will show that the PACM leads to inconsistencies when applied to real nuclei, while the SACM performs well.

The paper is structured as follows: in section $1 \mathrm{a}$ general discussion on phase transition, both in the PACM and SACM, is given, independent of a particular system. In Section III results are illustrated with two particular cluster systems, the $\alpha$-cluster states of ${ }^{20} \mathrm{Ne}$ and ${ }^{24} \mathrm{Mg}$. In the former, both clusters are spherical, while in the latter, one of them is deformed, leading to a more complex physical situation. Finally, in Section[V]conclusions are drawn and a discussion is presented on the differences between the PACM and the SACM and their importance in the study of nuclear clusters.

\section{PHASE TRANSITIONS IN ALGEBRAIC CLUSTER MODELS}

In what follows we apply the formalism developed in Paper I [1] to discuss phase transitions in the SACM and PACM. Before that we present the general framework within which the discussion will be implemented.

\section{A. Definition of a phase transition}

Phase transitions are investigated using the following steps and the recommendations of [10 12]. This method can be applied to any system and does not depend on the notion of symmetries. This presentation does not need the language of catastrophe theory [13], which simplifies considerations. 
a) In the first step the minima of the potential energy surface (PES) are determined in the space of the collective variables $\alpha_{m}$. In the present case there is only one relevant variable $\alpha$. This is due to the fact that the distance vector between the clusters can always be aligned along the intrinsic $z$-axis which connects the two clusters. The extrema are obtained from

$$
\frac{\mathrm{d} V}{\mathrm{~d} \alpha}=0
$$

This determines the position of the extrema at $\bar{\alpha}_{i}(i=1,2, .$.$) and the values V\left(\bar{\alpha}_{i}\right)$ of the potential there. The $\bar{\alpha}_{i}$ are the values of the variable $\alpha$ at the $i^{\text {th }}$ minimum, which is a function of the interaction parameters $\vec{p}=\left(p_{k}\right)$, with $p_{k}$ as a short-hand notation for the parameters $k=1,2, \ldots, n_{k}$, with $n_{k}$ being the number of parameters.

b) Once the extrema are obtained, one determines at each minimum the first and second derivatives of the potential with respect to the parameters of the model, i.e.,

$$
\frac{\partial^{n} V\left(\bar{\alpha}_{i}\right)}{\partial p_{k}^{n}}, n=1,2 .
$$

When we discuss the PACM and SACM further below, we will give first a general discussion on the properties of phase transitions, independent of the values of a particular subset of interaction parameters. In the subsequent concrete applications, however, we will fix most interaction parameters and vary only $x$ and $y$, which control the transition from one effective symmetry 14] to another. This will give us particular curves in the space of phase transitions.

c) The locations of phase transitions are determined by identifying the borders in the parameter space where at least two minima are at equal energy, i.e.,

$$
V\left(\bar{\alpha}_{i_{1}}\right)=V\left(\bar{\alpha}_{i_{2}}\right)
$$

for some index values $i_{1}$ and $i_{2}$ of the minima. This results in a relation

$$
f\left(p_{1}, \ldots, p_{n_{p}}\right)=0
$$

between the parameters, which allows one, in principle, to express one parameter in terms of the others (see the particular examples further below).

d) The phase transition is of order $m$ when, up to $n=$ $m-1$, the derivatives of the potential with respect to the free parameters are equal at the point of phase transition, while the $m^{\text {th }}$ derivative of the potential, with respect to its parameters, is discontinuous at the point of the phase transition, i.e.,

$$
\begin{aligned}
\frac{\partial^{n} V\left(\bar{\alpha}_{i_{1}}\right)}{\partial p_{k}^{n}} & =\frac{\partial^{n} V\left(\bar{\alpha}_{i_{2}}\right)}{\partial p_{k}^{n}} \quad, \quad \text { for } n<m \\
\frac{\partial^{m} V\left(\bar{\alpha}_{i_{1}}\right)}{\partial p_{k}^{m}} & \neq \frac{\partial^{m} V\left(\bar{\alpha}_{i_{2}}\right)}{\partial p_{k}^{m}} \quad
\end{aligned}
$$

Note again that this procedure for determining the order of a phase transition is quite general and does not depend on identifying the phase with a dynamical symmetry. Thus, this procedure can also be applied to systems that do not exhibit dynamical symmetries.

\section{B. A possible explanation for the differences in using coherent states and a numerical approach}

In the numerical calculations further below, we will see that, though the use of coherent states shows a clear phase transition, this is not the case in the numerical study, because the total number of bosons $N$ is fixed. In what follows, we indicate why this is so.

The main reason for using coherent states is that the phase transition is given by the crossing of two potential minima. In the case of a first-order phase transition, the two minima are well separated by a barrier and a simple crossing takes place. For the case of a secondorder phase transition, the spherical minimum coincides with the "deformed" one at $\alpha=0$ at a certain point in the parameter space. Once one minimum is deeper than the other one, an immediate jump by hand to the global minimum is made, using coherent states. This appears to produce the well defined phase transition. However, in numerical calculations within a finite system, the states are a mixture of states in both minima. Around the point of phase transition, the eigenstates of the Hamiltonian are a mixture of states in both minima. Only far away the states are located clearly in one or the other minimum. This produces, for finite $N$, a gradual change in the wave function and as a consequence a gradual change of the control parameters, like the energy or the expectation value of $\boldsymbol{n}_{\pi}$, which define the phase transition. For $N \rightarrow \infty$, the discontinuities in the derivatives of the potential will be clear cut.

As a consequence, the apparently clear phase transition for any number of $N$ is not a real one, because $N$ is finite. Nevertheless, the use of coherent states shows the points of phase transitions and of which order they will be for $N \rightarrow \infty$.

\section{Study of phase transitions in the SACM}

Paper I 1] contains the general expression of the Hamiltonian (in subsection 2.2) and the geometrically mapped SACM potential (in subsection 4.1). Here we recall only the essential formula, Eq. (30) of Paper I, 
necessary for the present discussion:

$$
\begin{aligned}
\langle\boldsymbol{H}\rangle= & \mathcal{C}(x, y)-(b+\bar{b}) x y\left(A(x, y) \alpha^{2} \frac{F_{11}\left(\alpha^{2}\right)}{F_{00}\left(\alpha^{2}\right)}\right. \\
& -B(x, y) \alpha^{4} \frac{F_{22}\left(\alpha^{2}\right)}{F_{00}\left(\alpha^{2}\right)}+\alpha^{6} \frac{F_{33}\left(\alpha^{2}\right)}{F_{00}\left(\alpha^{2}\right)} \\
& \left.-C(x, y) \alpha^{2} \frac{F_{20}^{N-2}\left(\alpha^{2}\right)}{F_{00}\left(\alpha^{2}\right)}\right)
\end{aligned}
$$

We also note that the $x=0$ case relevant to the $S O(4)$ to $S O(3)$ phase transition has to be discussed separately. (See Eqs. (35) to (38) in Paper I [1].)

\section{Phase transition diagram for the SACM: General considerations}

The complex structure of the geometrically mapped potential complicates an analytic treatment of the problem. It is therefore essential to formulate a set of criteria facilitating a straightforward way to determine the order of phase transitions.

Equation (6) demonstrates that the explicit dependence of the potential on the parameters $A, B$ and $C$ is linear of the type

$$
\widetilde{V}=\sum_{k} p_{k} \alpha^{m_{k}} f_{k}(\alpha)
$$

with $m_{k}>1$ and $p_{k}$ being a short-hand notation for the $k^{\text {th }}$ parameter. The $f_{k}$ are given by ratios of the functions $F_{p q}(\alpha)$ and are always greater than zero for $\alpha \neq 0$. Only the function $F_{20}^{N-2}$ approaches zero for $\alpha \rightarrow \infty$. (See Eq. (39) in Paper I [1].)

According to the general discussion on phase transitions in Subsection ПA let us now turn to the potential minima located at $\bar{\alpha}_{i}$. We investigate their dependence on the parameters $p_{k}$, standing for $A, B$ and $C$. The structure of (7) guarantees that $\bar{\alpha}_{1}=0$ is always an extremum. Furthermore, not only its first-order derivatives, but the function value is also zero at $\bar{\alpha}_{1}=0$. It is thus sufficient to focus on the second, deformed minimum, with $\bar{\alpha}_{2}$, for which the following consideration holds. There are two possibilities:

a) $\bar{\alpha}_{2}>0$, or

b) $\bar{\alpha}_{2}=0$.

The consequences are seen by determining the first absolute derivative of the potential with respect to the parameter $p_{k}$. This first derivative is given by

$$
\begin{aligned}
\frac{\mathrm{d} \widetilde{V}}{\mathrm{~d} p_{k}} & =\frac{\partial \widetilde{V}}{\partial p_{k}}+\frac{\partial \widetilde{V}}{\partial \bar{\alpha}_{i}} \frac{\partial \bar{\alpha}_{i}}{\partial p_{k}} \\
& =\frac{\partial \widetilde{V}}{\partial p_{k}}
\end{aligned}
$$

because $\frac{\partial \widetilde{V}}{\partial \bar{\alpha}_{i}}$ vanishes at the minimum. Taking into account (7), this is further expressed as

$$
\frac{\mathrm{d} \widetilde{V}}{\mathrm{~d} p_{k}}=\bar{\alpha}_{2}^{m_{k}} f_{k}\left(\bar{\alpha}_{2}\right)
$$

For case a) this expression is different from zero (remember that $\bar{\alpha}_{2}>0$ ), while the derivative within the spherical minimum is equal to zero. In other words, there is a first-order phase transition.

For case b), (9) is equal to zero, and therefore the phase transition must be of higher order. To determine which order, second-order derivatives are also needed:

$$
\begin{aligned}
\frac{\mathrm{d}^{2} \widetilde{V}}{\mathrm{~d} p_{k}^{2}} & =\frac{\mathrm{d}}{\mathrm{d} p_{k}}\left(\frac{\partial \widetilde{V}}{\partial p_{k}}\right) \\
& =\frac{\partial \bar{\alpha}_{2}^{m_{k}}}{\partial p_{k}} f_{k}\left(\bar{\alpha}_{2}\right)+\bar{\alpha}_{2}^{m_{k}} \frac{\partial f_{k}\left(\bar{\alpha}_{2}\right)}{\bar{\alpha}_{2}} \frac{\partial \bar{\alpha}_{2}}{\partial p_{k}} .
\end{aligned}
$$

Here we used the fact that in (9) there is no explicit dependence in $p_{k}$ left. Since in this case $\bar{\alpha}_{2}=0$, (10) reduces to

$$
\frac{\mathrm{d}^{2} \widetilde{V}}{\mathrm{~d} p_{k}^{2}}=\frac{\partial \bar{\alpha}_{2}^{m_{k}}}{\partial p_{k}} f_{k}\left(\bar{\alpha}_{2}\right)
$$

As both the $f_{k}$ functions and the partial derivatives are different from zero, so is (11) in general, i.e. the phase transition is of second order in case b).

In conclusion we have the simple identification of phase transitions:

a) When the deformed solution $\bar{\alpha}_{2}$ is different from zero, then there is a first-order phase transition.

b) When the deformed solution $\bar{\alpha}_{2}$ is equal zero, then there is a second-order phase transition.

\section{General discussion of phase transitions in the SACM}

In Fig. 1 the surface where a phase transition takes place is plotted in the space of the independent parameters $A, B$ and $C$. The solid line marks a change from first-order to second-order transitions. For larger, positive $C$ the transition is of second order, while for smaller, negative $C$ it is of first order. For completeness we show in Fig.2 the phase space diagram for the case when $x=0$, as it is the case for the $S O(4)$ to $S O(3)$ transition.

A remarkable finding is that at approximately $C \approx-15$ the surface of phase transition ceases to exist. Beyond that point, no phase transition can be observed, i.e., the straight dashed line in Fig. 1 at approximately $C \approx-15$ represents a critical line. This is shown by a dashed line in Fig. 1. One then can trace a straight line from below the surface, around the critical line, ending up above the surface without passing through a phase transition, which is similar to the critical point in the two-dimensional phase diagram of water. Fixing all interaction parameters except $x$ and $y$ (as we will do in 
the numerical applications), passing from one dynamical symmetry limit to another one will trace a line in this three-dimensional space. Depending on the fixed parameters, this line will or will not cross the surface of phase transition.

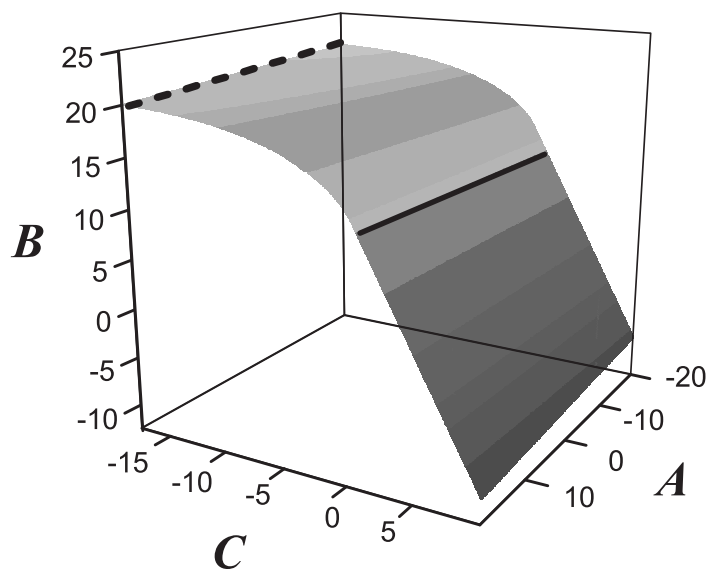

FIG. 1: The phase space diagram of the SACM as a function of the independent parameters $A, B$ and $C$. The solid line marks the change from a second- to a first-order phase transition, while the dashed line represents a 'critical line'.

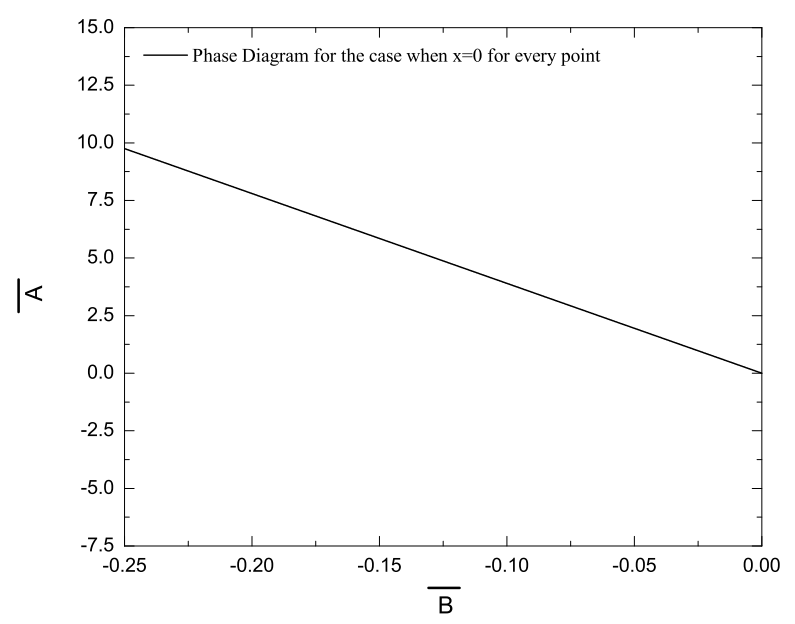

FIG. 2: The phase space diagram as a function of the independent parameters $\bar{A}$ and $\bar{B}$ for the $S O(4)$ to $S O(3)$ transition, within the SACM. As defined in Paper I, within the $S O(4)$ dynamical limit the $x=0$ and one has to define a new representation of the potential, involving the new parameters $\bar{A}$ and $\bar{B}$. (See also Paper I [1] for more.)

Figure 3 displays $\bar{\alpha}_{2}$ corresponding to the deformed solution at the point of phase transition as a function in $A$ and $C$. (Remember that the spherical solution corresponds to the always existing extremum at $\alpha=0$ in the case it is a local minimum.) Each point of the surface

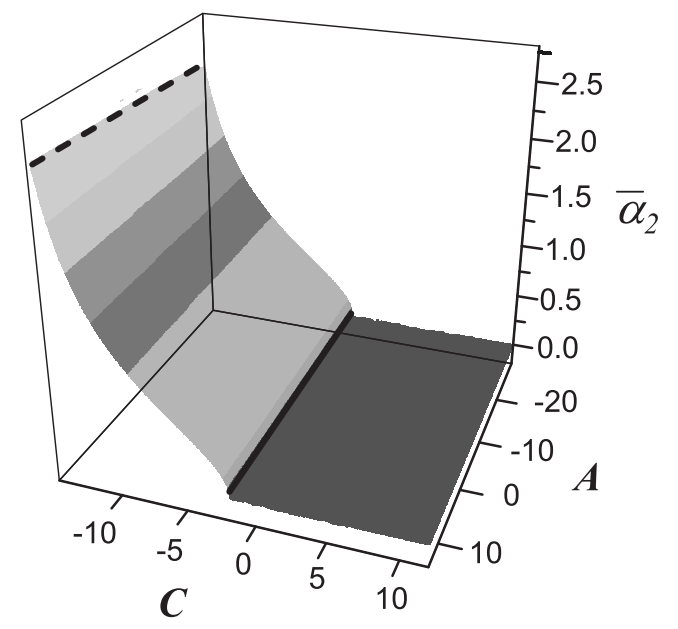

FIG. 3: The variable $\bar{\alpha}_{2}$ of the deformed solution, as a function in $A$ and $C . B$ is fixed by the requirement that one is at a point of a phase transition. The solid line marks the transition from a second- to a first-order phase transition, the dashed a 'critical line'.

represents also a given $B$ at which the phase transition occurs, i.e., $B$ is fixed by the requirement that there is a phase transition. In this figure the solid line also represents the change from one type of phase transition to the other one. For larger, positive $C \bar{\alpha}_{2}=0$ holds, and according to the discussion of subsection [IC 1, it corresponds to a second-order phase transition. For smaller, negative $C$ the $\bar{\alpha}_{2}>0$ holds at the point of phase transition, i.e., it corresponds to a first-order phase transition.

The whole phase structure of the system is illustrated in Fig. 4 which corresponds to the fixed value $A=10$. The solid line represents a cut through the phase transition surface. For $C>0$ the phase transition is of second order, while for $C<0$ is of first order. The Roman numbers indicate the following regions: i) Region I corresponds to the existence of two minima (one spherical and the other deformed), with the deformed one as the global minimum. ii) Region II also corresponds to two minima with the spherical as the global one. iii) In region III there is only one spherical minimum while iv) in region IV there is only one deformed minimum. The region denoted by a zero refers to potential with no minimum. The other dashed lines do not indicate phase transitions, rather they separate the areas where two minima exist and the ones where only one minimum exists. The line of phase transition ends at approximately $C \approx-15$, corresponding in this case to a point.

There is another point on the solid line, where the first-order phase transition turns over into a second-order phase transition. A distinction has to be made between this and the critical point of the phase diagram of water, where before the critical point the phase transition is of first order, while at the critical point it is of second order. In our case, however, the phase transition does not end at this point, rather it continues as second-order 
one in the domain with $C>0$. One can now draw a curve which encircles this line, going from a spherical phase to a deformed one. The value of $C$ at which this phenomenon happens, varies with $A$ and $B$.

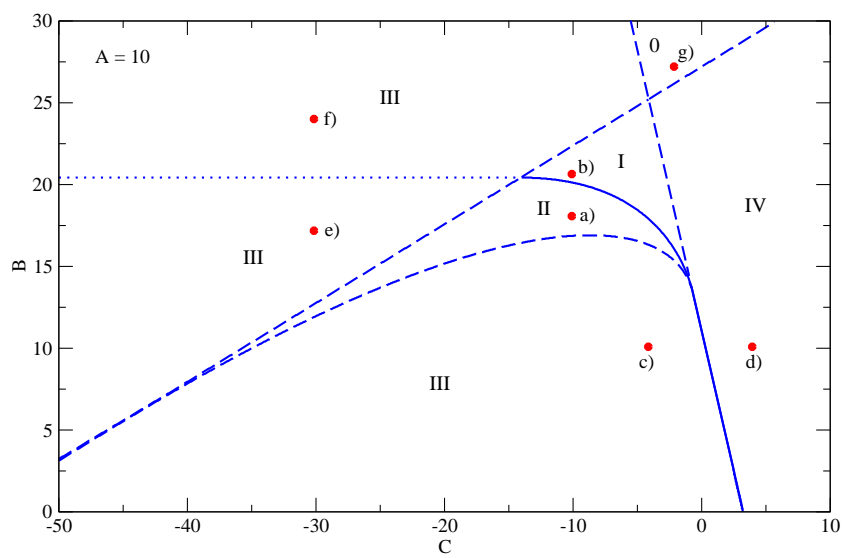

FIG. 4: (color online). A cut through the phase at $A=10$. The notation is explained in the text.
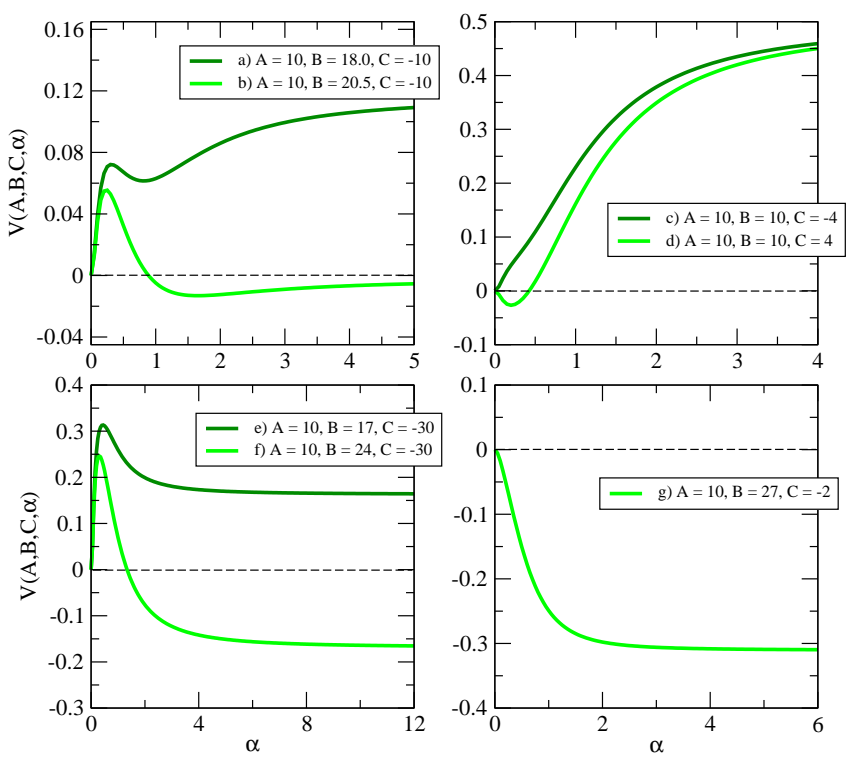

FIG. 5: (color online). Several potentials for different values of $B$ and $C$, for $A=10$ are shown. Some potentials approach a positive value, i.e., for $N \rightarrow \infty$ they goes to $+\infty$. Others approach a negative value, i.e., for $N \rightarrow \infty$ they approach $-\infty$

The horizontal dotted line represents a division (see discussion in $\amalg \mathrm{A}$ ): above this line the potential approaches a negative infinite value for $N \rightarrow \infty$, i.e., the potential gets unstable and the cluster system dissolves, once getting to the "deformed" solution. Below that line the potential approaches plus infinity for $N \rightarrow \infty$, thus the deformed solution corresponds to a stable cluster system.

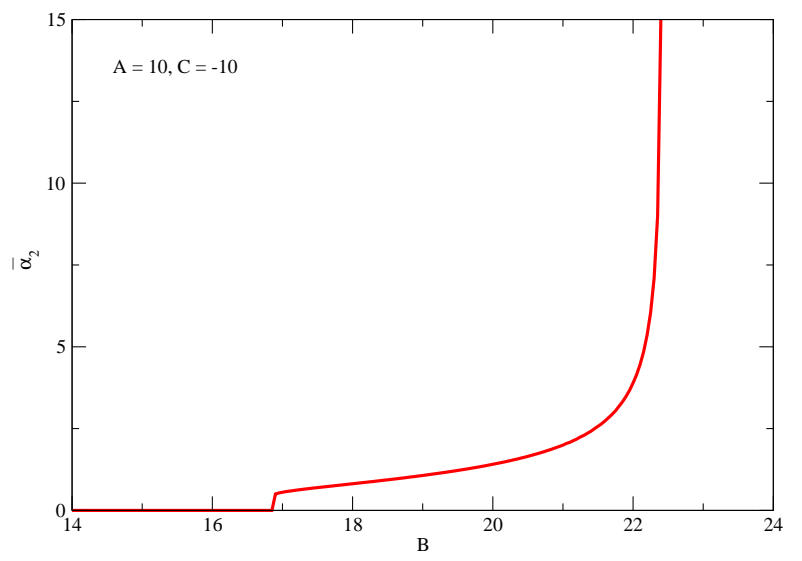

FIG. 6: (color online). The value of $\bar{\alpha}_{2}$ at $A=10$ and $C=-10$ as $B$ moves through regions.

Fig. 5 gives a sample of energy functionals at a fixed $N\left(n_{0}=8\right.$ and $\left.N+n_{0}=20\right)$ in different regions, the specific points being marked with lower-case letters in Fig. (4. The upper left panel shows functionals either side of the first-order phase transitions, points a) and b). The upper right one shows graphs either side of the secondorder phase transitions, points c) and d). The lower left panel shows a functional either side of the dotted line in the Region III of greater $B$, points e) and f), showing that while each have a barrier, one is bound as $\alpha \rightarrow \infty$, and the other is unbound. These tails are dependent on $N$, with the unbound approaching infinity and the bound negative infinity as $N \rightarrow \infty$. Thus, this region is unphysical. The Region III of lesser $B$, shown in the second panel, has no barrier, and is bound as $\alpha \rightarrow \infty$. The fourth panel shows a fully unbound potential with no minima from Region 0, point $\mathrm{g}$ ).

For completeness, Fig. 6 traces the value of $\bar{\alpha}_{2}$ for $14<B<24$ at $C=-10$ in Fig. 4, which thus represents a movement between several regions in that figure (and is therefore analogous to being a subset of Fig. (3)). It shows that the critical point corresponds to $\bar{\alpha}_{2}$ asymptotically approaching $\infty$ at $B$ slightly greater than 22 .

Note that the discussion of phase transitions is completely independent of the system considered, which is of great advantage. In the next section we will consider particular systems and study the properties of their phase transitions.

\section{The PACM case: Pauli principle not taken into account}

Here we apply the results obtained in subsection 4.2 of Paper I 1]. We display only the expression of the normalized potential in Eq. (46) of Paper I:

$$
\widetilde{V}=\left\{A \beta^{2}-B \beta^{4}+\beta^{6}\right\}
$$


We also remind the reader that the $S O(4)$ to $S O(3)$ transition needs a separate discussion, because in that case the potential reduces to a quartic one. (See the discussion in subsection 4.2 of Paper I [1].)

\section{Phase transitions}

The extrema are found by setting the first derivative with respect to $\beta$ equal to zero. We obtain, in general, two solutions

$$
\text { solution } 1: \quad \beta_{1}=0 \text {, }
$$

noting that in this case the potential value is always $\widetilde{V}\left(\beta_{1}\right)=0$, and

$$
\text { solution } 2: \quad \beta_{2}^{2}=\frac{1}{3}\left(B \pm \sqrt{B^{2}-3 A}\right),
$$

where the value of the potential at the extremum with the positive sign is given by

$$
\begin{aligned}
\widetilde{V}\left(\beta_{2}\right)=\frac{1}{27}( & \left.B+\sqrt{B^{2}-3 A}\right) \\
& \times\left(6 A-B^{2}-B \sqrt{B^{2}-3 A}\right) .
\end{aligned}
$$

The barrier maximum is obtained by choosing the negative sign in front of the square root expressions. The last equation is acceptable only when the solution in (14) is real and positive. The solution is real for $A \leq B^{2} / 3$. When $B<0$ and $A>0$ holds, no deformed solution exist. In fact, inspecting Eq. (12) for $A>0$ there is always a minimum at $\beta=0$, while a negative $B$ always gives a positive contribution, $+\beta^{4}$. When $A$ and $B$ are negative, the potential is negative at small $\beta$ and is turned over by the positive contributions $\sim+\beta^{4}$ and $+\beta^{6}$. In this case, only a deformed minimum exists.

The structure explained above is summarized in Fig. 7 The horizontal line is the $B$ axis while the vertical one is the $A$ axis. On the left hand side $(B<0)$ a spherical minimum exists for $A>0$, while for $A<0$ the minimum is deformed. The phase transition takes place at the line $A=0$. This will be discussed further below as case $\mathrm{b}$ ). For $B>0$ and $A<0$, a deformed minimum always exists. For $A>0$ and below the dashed curve $A=B^{2} / 3$ a deformed minimum coexists with a spherical minimum until $A=0$ is reached. The solid curve is determined by requiring that the potential minima at $\beta_{1}$ and $\beta_{2}$ are degenerate, i.e.,

$$
\widetilde{V}\left(\beta_{1}\right)=\widetilde{V}\left(\beta_{2}\right),
$$

which leads to the condition

$$
A=\frac{B^{2}}{4} .
$$

Between the upper dashed and the solid curves the spherical minimum is the global one, while below the solid

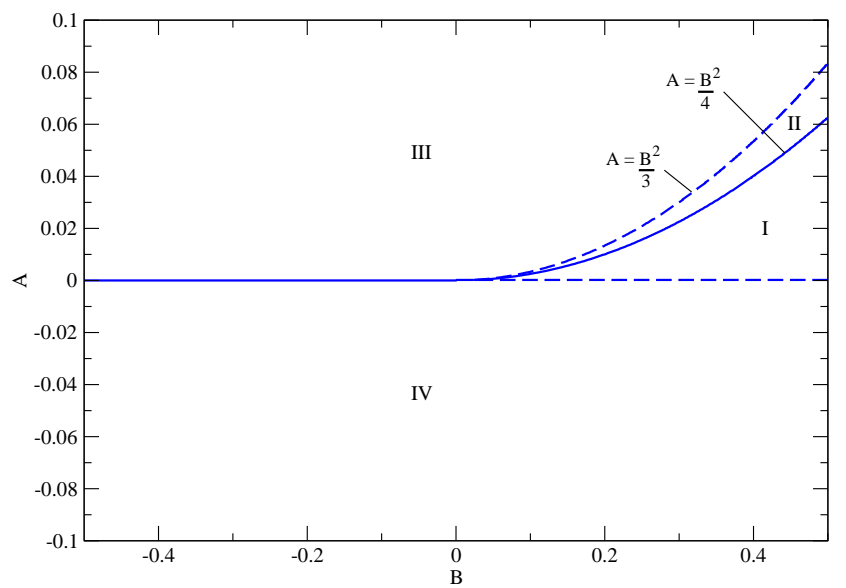

FIG. 7: (color online). The parameter phase diagram for the PACM [15, 16]. The horizontal axis is $B$, while the vertical axis corresponds to $A$. In Region I and Region II two minima exist, one spherical and one deformed. In Region I the global minimum is the deformed one, while in Region II it is the spherical minimum. In Region III only a spherical minimum exists and in Region IV the only minimum is a deformed one.

curve the global minimum is the deformed one. Crossing the solid line a phase transition takes place, which will be called below case a). It is of no surprise that the phase diagram in Fig. [7] is similar to Fig. 2 of [17].

Case a), $B>0$ :

The potential is exactly zero at $\beta_{1}=0$, and thus

$$
\frac{\partial^{n} \widetilde{V}\left(\beta_{1}=0\right)}{\partial A^{n}}=0
$$

for all $n \geq 0$. Contrary to this, the first derivative of the potential at $\beta_{2}$, the deformed minimum, taken at $A=B^{2} / 4$ where the height of the deformed minimum equals that of the spherical one, is given by

$$
\frac{\partial \widetilde{V}\left(\beta_{2}\right)}{\partial A}=\frac{1}{2} B
$$

which is clearly different from zero for $B>0$. Thus the phase transition is of first order for $B>0$.

We can repeat the calculation for the derivatives in $B$, leading to the same conclusion. Also, any combination of curves given by the variable $(c A-s B$ ) (with $c=\cos \phi$ and $s=\sin \phi$, with $\phi$ as the angle between the tilted straight line and the $A$ axis) leads to the same conclusion.

Case b): $B \leq 0$

We will vary $A$ from positive to negative values for a fixed $B$. The same steps as taken for case a) can be applied, setting $B=-|B|$. The first and second derivatives 
of the potential with respect to $A$ are given by

$$
\begin{aligned}
\frac{\partial \widetilde{V}\left(\beta_{2}\right)}{\partial A} & =\frac{1}{3} \sqrt{B^{2}-3 A}\left(1-\frac{|B|}{\sqrt{B^{2}-3 A}}\right) \\
\frac{\partial^{2} \widetilde{V}\left(\beta_{2}\right)}{\partial A^{2}} & =-\frac{1}{2 \sqrt{B^{2}-3 A}} .
\end{aligned}
$$

Evaluating these derivatives at $A=0$ and $B=-|B|$ gives

$$
\frac{\partial \widetilde{V}}{\partial A}=0 \quad, \quad \frac{\partial^{2} \widetilde{V}}{\partial A^{2}}=-\frac{1}{2 B}
$$

For this case we also have to determine the derivatives with respect to $B$, given at the point of phase transition, i.e., setting $A=0$ afterward, and $B=-|B|$,

$$
\begin{aligned}
\frac{\partial \widetilde{V}}{\partial B} & =-\frac{1}{9}\left[-|B|+\sqrt{|B|^{2}-3 A}\right]^{2}=0 \\
\frac{\partial^{2} \widetilde{V}}{\partial B^{2}} & =-\frac{2}{9}\left[-|B|+\sqrt{|B|^{2}-3 A}\right]\left[1-\frac{|B|}{\sqrt{|B|^{2}-3 A}}\right] \\
& =0 .
\end{aligned}
$$

Changing $B$ corresponds only to moving on the line of phase transition, which does not make sense, because one has to cross the line of phase transition. However, a combination of changing $A$ and $B$ corresponds to an inclined straight line, crossing the line of phase transition. This means that one has to consider a general line given by the variable $C=c A-s B$, with arbitrary $c=\cos \phi$ and $s=\sin \phi$, with $\phi$ being an arbitrary rotation angle. Determining at the point of phase transition the first- and second-order derivatives of $\widetilde{V}$ with respect to $C$ leads to

$$
\begin{gathered}
\frac{\partial \widetilde{V}}{\partial C} \rightarrow c \frac{\partial \widetilde{V}}{\partial A} \\
\frac{\partial^{2} \widetilde{V}}{\partial C^{2}} \rightarrow c^{2} \frac{\partial^{2} \widetilde{V}}{\partial A^{2}}
\end{gathered}
$$

Because the first derivative of the potential with respect to $A$ is zero, while the second is different from zero at the point of phase transition, we can claim also that the first derivative with respect to $C$ is zero, while the second is different from zero. We conclude that the phase transition is of second order.

\section{NUMERICAL STUDIES}

This section deals with two widely known cluster systems: ${ }^{20} \mathrm{Ne}$ as ${ }^{16} \mathrm{O}+\alpha$, where both clusters are spherical, and ${ }^{24} \mathrm{Mg}$ as ${ }^{20} \mathrm{Ne}+\alpha$, where one of them is deformed.

Since our aim is studying transitions from one particular dynamical symmetry limit to another one, we first fix all the interaction parameters except $x$ and $y$. The $\hbar \omega$ parameter was not fixed, but rather it was chosen according to the harmonic oscillator constant corresponding to the unified nucleus. The fixed interaction parameters are determined in such a way that in the dynamical symmetry limits the spectrum appears with the same scale as the physical measured one. The spectrum of the real nucleus would probably correspond to a single point in the $(x, y)$ parameter space. However, our aim is not reproducing the exact spectrum, rather to reach a conceptual understanding of phase transitions when going from one dynamical symmetry to another, and investigating the conjecture that a phase is defined by an effective symmetry. One alternative method would be to adjust several cluster systems and to try to find a series of systems which, for example, would cross the surface of phase transitions at one point. We do not follow this way, rather postponing it for later consideration.

\section{A. Two spherical clusters: ${ }^{16} \mathrm{O}+\alpha \rightarrow{ }^{20} \mathrm{Ne}$}

In this case the only degree of freedom is the radial motion, as the clusters do not have an internal structure apart from the fact that they are composed of fermions, which have to obey the Pauli exclusion principle. Therefore the cluster representation is $\left(\lambda_{C}, \mu_{C}\right)=(0,0)$. As described in Paper I [1], the $S O(3)$ limit does not exist as an independent limit in this case (the $S O(3)$ Hamiltonian is a reduced version of the the $S U(3)$ Hamiltonian), so the $y=1$ choice has to be made. The only transition to consider is thus between the $S U(3)$ and the $S O(4)$ limits.

Concerning the determination of the parameters, one has to take into account that some parameters appear in both dynamical symmetry limits, like $\gamma$ preceding $\boldsymbol{L}^{2}$ (see Eqs. (10) and (11) in Paper I [1]). We first determine the parameters in the $S U(3)$ dynamical symmetry limit, which fixes $\gamma$, and then we determine the remaining parameter $c$, which appears in the $S O(4)$ dynamical symmetry limit. The terms $\mathcal{C}_{2}\left(\lambda_{C}, \mu_{C}\right)$ and $\boldsymbol{L}_{C}^{2}$ do not contribute to the Hamiltonian in this case, so the corresponding parameters are kept zero. Note that in this case $\boldsymbol{L}_{R}^{2}=\boldsymbol{L}^{2}$.

\section{The $S A C M$}

In the first step the parameters are adjusted within the $S U(3)$ limit, setting $x=1$ and $y=1$ and in the $S O(4)$ limit, setting $x=0$ and $y=1$. The parameters are depicted in Table 1 . The SACM yields reasonable results, because the ground-state band belongs to $n_{\pi}=8$, $(\lambda, \mu)=(8,0)$, where $n_{\pi}=8$ corresponds to the minimal number of relative oscillation quanta $n_{0}$ required by the Wildermuth condition. The first excited $0^{+}$state corresponds to a $2 \hbar \omega$ excitation and naturally lies at high energy as required by the experimental data. The spectra in the $S O(4)$ and $S U(3)$ limits are shown in the left and 
TABLE I: Parameter values defining the ${ }^{16} \mathrm{O}+\alpha$ interaction. See Eq. (11) in Paper I [1].

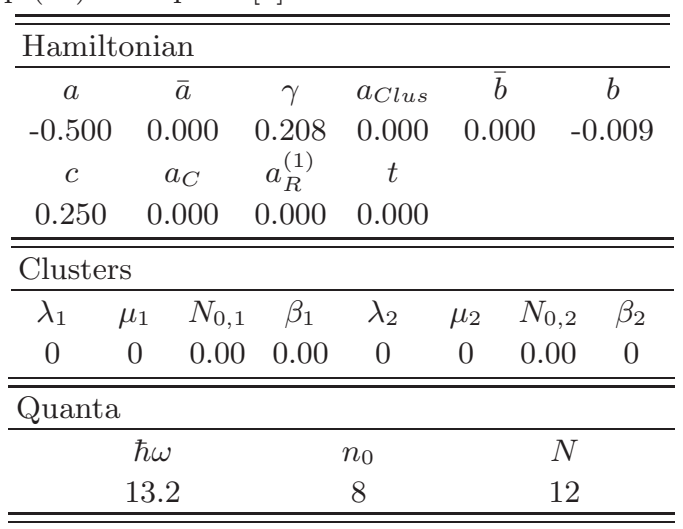

right extreme of right panel of Fig. 11, respectively. The spectrum of experimental ${ }^{20} \mathrm{Ne}$ states, each corresponding to this clusterisation, is shown in the left panel. As already mentioned, the real nucleus will lie somewhere between $x=1$ and $x=0$, though, the fit at $x=1$ is acceptable.

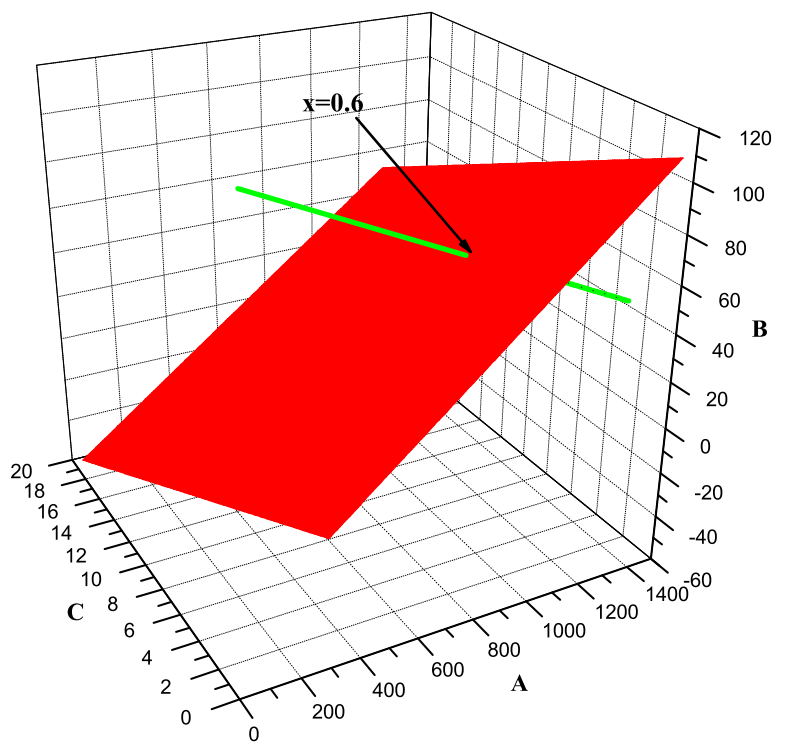

FIG. 8: (color online). Part of the SACM phase space diagram, where the curve of the parameter values, as a function of $x$, crosses the surface of phase transition. The crossing occurs at approximately $x=0.6$ and happens at the surface related to the second-order phase transition.

In order to see if a phase transition appears, and of which order it is, we added a curve to the $(A, B, C)$ phase space in Fig. 8 depicting the transition from $x=1$ to $x=0$. The figure shows only the relevant part of the phase space, i.e. the one where the curve crosses the surface associated to the second-order phase transition. This occurs at approximately the $x=0.6$ parameter value. The actual values of $A, B$ and $C$ associated with this $x$ can be seen in Fig. 9, In summary, the situation in this example corresponds to a phase transition of second order.

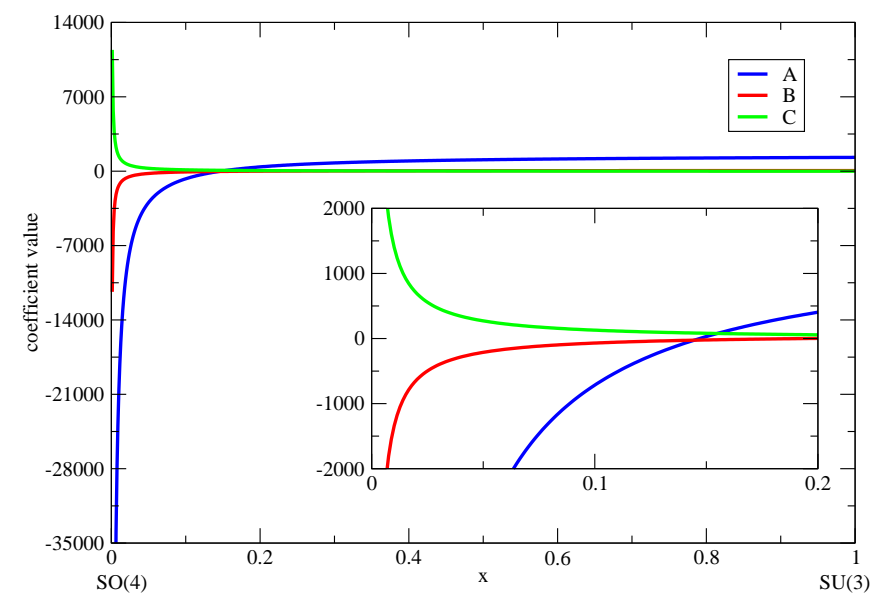

FIG. 9: (color online). The parameters $A, B$ and $C$ as a function of $x$, considering the $S U(3)$ to $S O(4)$ transition. The inset shows a region of large change in $A, B$ and $C$ with change in $x$. A selected range of values is shown in the inset, for a better reading.

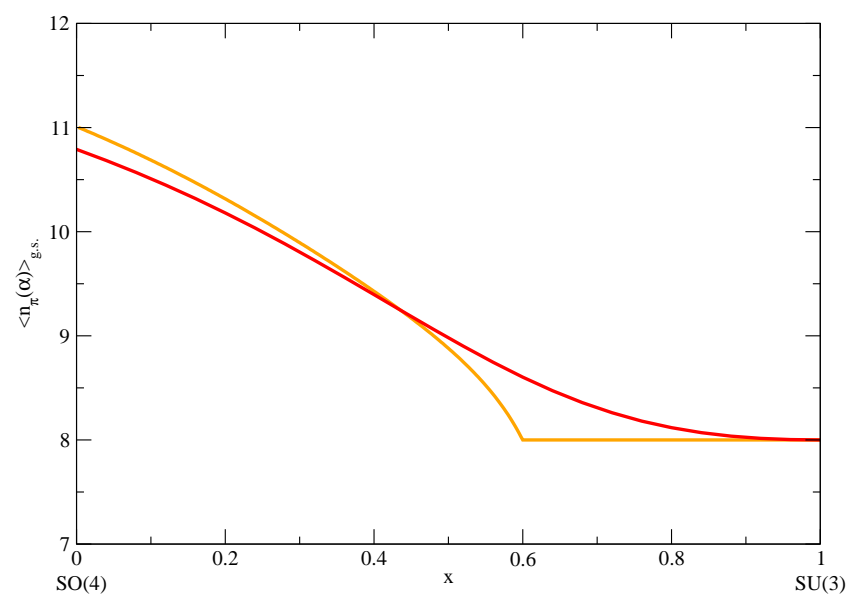

FIG. 10: (color online). The expectation value of $n_{\pi}$ (vertical axis) as the function of $x$. Starting from $x=1$ the expectation value is 8 , given by the lowest possible number of $\pi$ quanta. From $x=0.6$ on we observe a rise in the expectation value, reaching $n_{\pi}=11$ at $x=0$. The phase transition takes place at $x=0.6$.

Figure 10 displays how the expectation value of $\boldsymbol{n}_{\pi}$ changes as the function of $x$. The lighter (yellow online) and darker (red online) curves depict the result of the geometrical mapping and the numerical diagonalization, respectively. From $x=1$ up to the point of phase transition, the effective $S U(3)$ limit is realized and the expectation value is equal to the minimal number of $\pi$ bosons, i.e., $n_{0}=8$. Below $x=0.6$ the darker (red) 


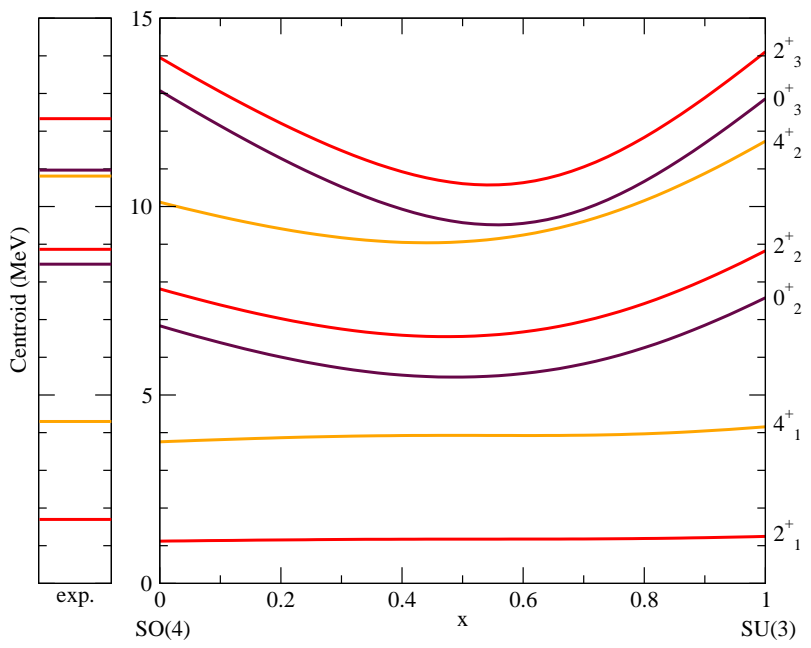

FIG. 11: (color online). The lowest energy states as the function of $x$ for ${ }^{16} \mathrm{O}+\alpha \rightarrow{ }^{20} \mathrm{Ne}$. The spins and parities are depicted in the legend.

curve begins to rise slightly, indicating that the structure of the system is changing.

The right panel of Figure 11 shows the lowest energy states of this clusterisation, those fitted, as the function of $x$. A smooth behavior is observed, with an accumulation of states near $x=0.6$. This is in accordance with the expectation value of $\boldsymbol{n}_{\pi}$ in Figure 10.

\section{The PACM}

This type of model was considered in [18], where a pure schematic investigation on possible phase transitions was presented. The model space was restricted to low $n_{\pi}$ excitations. No parameter fit was applied to a physical system. We show here that the relation to a physical system is of utmost importance and can discriminate between physical and non-physical models.

As a first step, we tried to adjust the parameters of the model in the $S U(3)$ limit $(x=1$ and $y=1)$ and in the $S O(4)$ limit $(x=0$ and $y=1)$. However, we already encountered severe problems in the $S U(3)$ limit: The model space for even angular momentum starts with $n_{\pi}=0$, thus the lowest states are comprised by $n_{\pi}=0$, $(\lambda, \mu)=(0,0)$, which contains one $J^{\pi}=0^{+}$state, and by $n_{\pi}=2,(\lambda, \mu)=(2,0)$, which contains a $0^{+}$and a $2^{+}$ state. The problems encountered are:

i) Since the factor in front of $\boldsymbol{L}^{2}$ has to be positive and supposing that the ground state belongs to $n_{\pi}=0$ and the next excited positive parity state belongs to $n_{\pi}=2$, the first excited $0^{+}$state will always be lower than the first excited $2^{+}$state. This contradicts the experimental spectrum with $E\left(2_{1}^{+}\right)=1.634 \mathrm{MeV}$ and $E\left(0_{2}^{+}\right)=8.7 \mathrm{MeV}$.

ii) Since the $2_{1}^{+}$state belongs to a $2 \hbar \omega$ excitation, with $\hbar \omega=13.2 \mathrm{MeV}$, the quadrupole-quadrupole interaction has to be unnaturally strong in order to shift the energy to $1.634 \mathrm{MeV}$. This, in turn, will move very high $n_{\pi}$ excitations to low energy, even below the supposed ground state with $n_{\pi}=0$.

iii) Due to the completely different $S U(3)$ structure of the states within the ground state "band", one can not talk about a rotation band.

Restricting to the $S U(3)$ limit, the eigenvalues of the Hamiltonian are given by

$$
E=\hbar \omega n_{\pi}+\left(a-b n_{\pi}\right) n_{\pi}\left(n_{\pi}+3\right)+\gamma L(L+1) \quad .
$$

Here we already see that for $\gamma>0$ and a fixed $n_{\pi}$, higher spin states are higher in energy. In order to adjust the $2_{1}^{+}$and the $0_{2}^{+}$states to the experimental energies, the fitting routine assigns to both the ground state and the $2_{1}^{+}$state a different $n_{\pi}$. Using $N=20$, as a result we obtain $n_{\pi}=20$ (the total number of bosons was set to be 20) for the $0_{1}^{+}$and the $2_{1}^{+}$state, while the $0_{2}^{+}$state belongs to $n_{\pi}=0$. When we change the total number of bosons, we get similar results. The mere fact that we have to involve states with $n_{\pi}=N$ indicates that no convergence is achieved, considering that $N$ represents a cut-off value.

Similar results are also obtained when the $S O(4)$ limit is considered. In the $S O(4)$ limit, the energy is given by

$$
E=\frac{c}{4}(N-\omega)(N+\omega+2)+\gamma L(L+1),
$$

where $\omega$ refers now to the $S O(4)$ quantum number with $\omega$ $=N, N-2, \ldots, 0$ or 1 . The lowest state is normally taken as $\omega=N$, which contains $L=0,1, \ldots, \omega$. Thus, choosing $N=20$, the ground-state band $(\omega=20)$ is composed of the angular momentum states $L=0,1, \ldots, 20$ and the first excited band with even spin $(\omega=18)$ is given by the states $L=0,1, \ldots, 18$. Thus, the first excited $0^{+}$state can be set at higher energies than the first excited $2^{+}$ state, adjusting the parameter $c$. Everything seems to be in order, except for the problems which the following discussion demonstrates.

The difference with respect to the $S U(3)$ limit is that a state is a mixture of many basis states in $S U(3)$. We adjusted the spectrum of ${ }^{20} \mathrm{Ne}$ to the $S O(4)$ limit and confirmed that for $L=0$ the expectation value of the operator $\boldsymbol{n}_{\pi}$ is given by [19]

$$
\left\langle\boldsymbol{n}_{\pi}\right\rangle=\frac{N-1}{2} .
$$

The problem here is that when the cut-off is increased, the structure of the states change: $\left\langle\boldsymbol{n}_{\pi}\right\rangle$ increases, implying that no convergence has been reached. This also implies high shell excitations, if we assume that the two clusters are moving in a shell model mean field, which has been proven in many microscopic calculations [20]. In the PACM, however, the mean field $\hbar \omega \boldsymbol{n}_{\pi}$ has no specific meaning, i.e., the parameter $\hbar \omega \rightarrow d$ can be very small. As shown above, the spectrum can be easily adjusted within the $S O(4)$ limit. The fact that it adjusts 
TABLE II: Parameter values for the ${ }^{20} \mathrm{Ne}+\alpha$ interaction. See Eq. (11) in Paper I [1].

\begin{tabular}{|c|c|c|c|c|c|c|}
\hline \multicolumn{7}{|c|}{ Hamiltonian } \\
\hline$a$ & & $\bar{a}$ & $\gamma$ & $a_{\text {Clus }}$ & $\bar{b}$ & $b$ \\
\hline-1.396 & & 136 & 0.197 & 0.000 & -0.116 & 0.045 \\
\hline$c$ & & $u_{C}$ & $a_{R}^{(1)}$ & $t$ & & \\
\hline 0.470 & & 079 & 0.053 & 0.664 & & \\
\hline \multicolumn{7}{|c|}{ Clusters } \\
\hline$\lambda_{1}$ & $\mu_{1}$ & $N_{0,1}$ & $\beta_{1}$ & $\lambda_{2}$ & $N_{0,2}$ & $\beta_{2}$ \\
\hline 8 & 0 & 48.5 & 0.73 & 0 & 4.5 & 0 \\
\hline \multicolumn{7}{|c|}{ Quanta } \\
\hline \multicolumn{3}{|c|}{$\hbar \omega$} & \multicolumn{2}{|r|}{$n_{0}$} & \multicolumn{2}{|c|}{$N$} \\
\hline \multicolumn{4}{|c|}{12.6} & 8 & \multicolumn{2}{|c|}{12} \\
\hline
\end{tabular}

the spectrum suggests that there must be some truth in it. Nevertheless, the basic degrees of freedom (clusters plus relative motion) cannot be interpreted as real clusters or relative motion, as is done in microscopic cluster studies, but must be in a complicated relation with them. Just what relations these are remains a big problem.

This result demonstrates that the model shows inconsistencies and the reasons are exposed in the arguments i) and ii) above. The interpretational problems of the PACM have already been indicated in [14].

A possible solution to this problem is to redefine the pairing operator as $\left[\left(\boldsymbol{\pi}^{\dagger} \cdot \boldsymbol{\pi}^{\dagger}\right)-R^{2}\left(\sigma^{\dagger}\right)^{2}\right]$, i.e., the introduction of a new parameter $R^{2}$ which can be set proportional to $1 / N$. In this way, the $N$ dependence can be eliminated and physical results can be expected. This procedure was adopted in [21], where the vibron model was extended to three clusters describing the ${ }^{12} \mathrm{C}$ nucleus as an oblate symmetric top. Though, in [21] the $S O(7)$ limit in a $U(7)$ algebraic model, which describes three clusters, is considered, the same ideas can be translated here.

\section{B. One spherical and one deformed cluster: ${ }^{20} \mathrm{Ne}+\alpha \rightarrow{ }^{24} \mathrm{Mg}$}

This is the first example where the cluster part has a structure owing to the deformed ${ }^{20} \mathrm{Ne}$.

\section{The $S A C M$}

In order to analyze the transitions between the $S U(3)$, $S O(4)$ and $S O(3)$ limits, first the parameters were fixed in the three limits. The results are displayed in Table II

Figures 12 and 13 display the expectation value of $\boldsymbol{n}_{\pi}$ and the lowest states in energy, respectively, for all three transitions. The darker (red online) curve in Fig. 12 shows the results of the numerical diagonalization, while the lighter (yellow online) curve shows the one of the

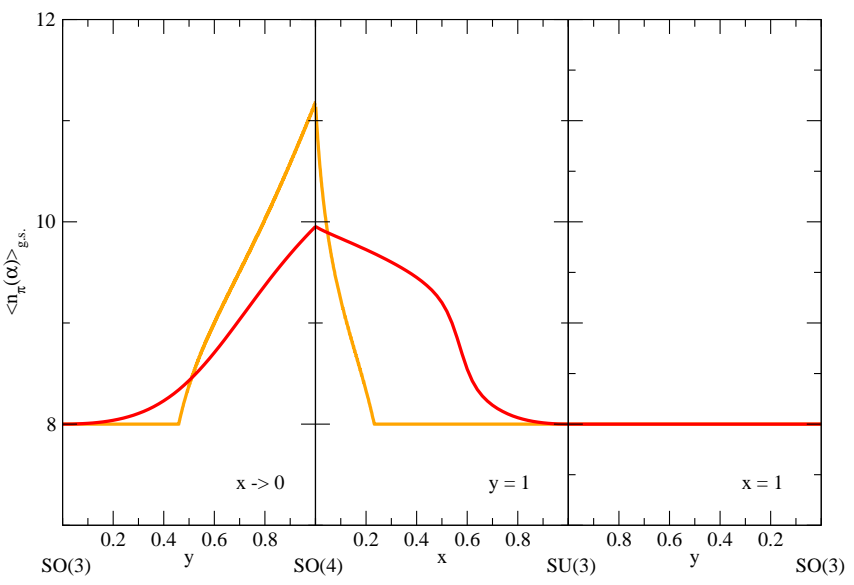

FIG. 12: (color online). Expectation value of $\boldsymbol{n}_{\pi}$ for the three transitions i) $S U(3)$ to $S O(4)$, ii) $S U(3)$ to $S O(3)$ and iii) $S O(4)$ to $S O(3)$.

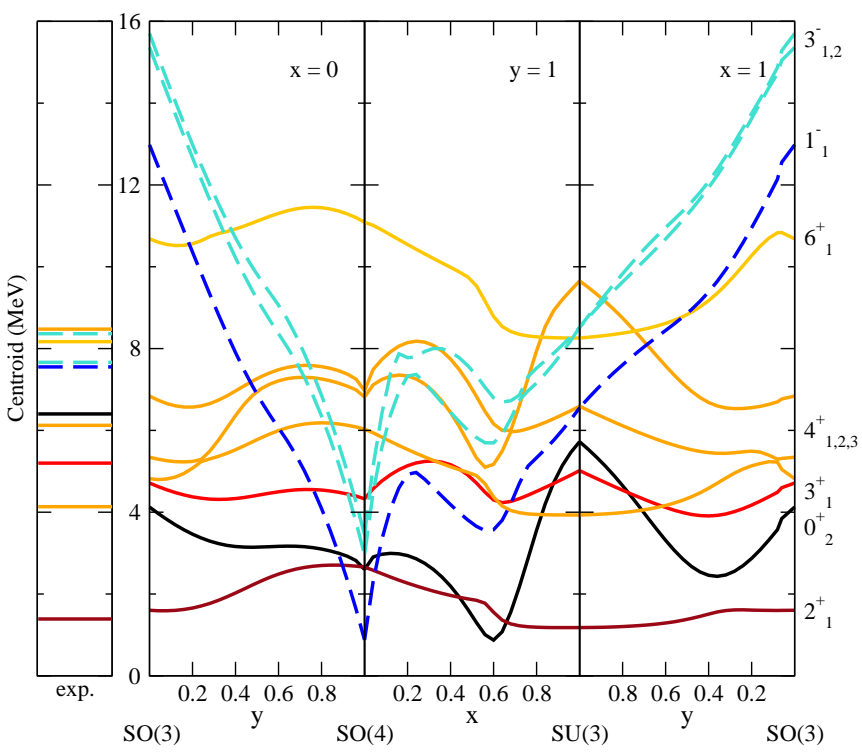

FIG. 13: (color online). The lowest states in the three transition i) $S U(3)$ to $S O(4)$, ii) $S U(3)$ to $S O(3)$ and iii) $S O(4)$ to $S O(3)$.

geometrical mapping. The results are qualitatively similar to those encountered in the former example. The expectation value of $\boldsymbol{n}_{\pi}$ starts in the $S U(3)$ limit at 8 and increases towards the $S O(4)$ limit. The transition is smooth in the numerical calculation, while in the geometrical mapping the transition is pronounced well. The transition is indicated by a sudden change in the slope at above $x=0.2$ in the central panel of Fig. 12. The energy spectrum in Figure 13 does not show a particular structure at points of phase transition. Therein, the leftmost panel is again the experimental spectrum used, and the right three are theoretical results as $x$ and $y$ are adjusted. In the $S U(3)$ to $S O(3)$ transition no phase change 
appears, because the global minimum of the potential is always at $\alpha=0$. One can observe a distinct behavior as a function of $x$ and $y$ between the states with positive and negative parity, marked as solid and dashed curves, respectively. The latter are more sensitive to the change in $x$ and $y$.

Similar properties can be observed in the other limits, i.e., see the leftmost panel in Fig. 12, In the rightmost panel of Fig. 12 no phase transition occurs. The energy spectrum also shows an accumulation of states at low energy at the point of phase transition.

\section{The PACM}

Here we find similar inconsistencies with respect to the model space as in the case of ${ }^{20} \mathrm{Ne}$ in 1 A 2. The cluster irrep of ${ }^{20} \mathrm{Ne}$ is $(8,0)$, while the relative oscillation irreps are $\left(n_{\pi}, 0\right)$, with $n_{\pi}=0,1,2, \ldots$ Restricting to positiveparity states only, the lowest energy model space, in the $S U(3)$ limit, consists of $(8,0)$ at $0 \hbar \omega$, and $(10,0)$ and $(6,2)$ for the $2 \hbar \omega$ excitation. The ground-state band is a $(8,0)$ irrep and the lowest $K=2$ band is the $(10,0)$ irrep at $2 \hbar \omega$. Even if we change $\hbar \omega$ to an arbitrary small parameter $a_{1}$, in order to bring the $2 \hbar \omega$ states down in energy, the internal structure of the ground state band is not what we expected, namely $(8,4)$. Also there is no $\mathrm{B}(E 2)$ transition between the ground state band and the $K=2$ band, because they belong to different irreps. (See the discussion in Subsection 2.1 in Paper I [1].)

For the $S O(4)$ limit one obtains a satisfactory fit, but again with the fact that the expectation value of the number operator $\boldsymbol{n}_{\pi}$ depends on the cut-off $N$.

For these reasons, we do not present figures of the spectra and expectation values.

\section{CONCLUSIONS}

Phase transitions were investigated in two algebraic cluster models, one of which observed the Pauli exclusion principle between the nucleons of the individual clusters (SACM), while the other (PACM) did not. This analysis was based on the results of a previous work 1], in which the geometric mapping of the two models had been per- formed using the coherent state formalism, leading to appropriate potential energy surfaces. The dynamical symmetries of the SACM and PACM had also been identified in [1], and in the present analysis special attention was paid to transitions between phases associated with the $S U(3), S O(3)$ and $S O(4)$ dynamical symmetries. The potential energy surfaces depended on the parameters appearing in the Hamiltonian shared by the SACM and PACM, including also the $x$ and $y$ variables controlling the transitions between the three dynamical symmetries. The phase space was reparameterized in terms of three parameters $A, B$ and $C$.

In the case of the PACM, the potential energy surface was a sextic oscillator in the intercluster distance variable, while for the SACM the potential shape was more complex due to the restrictions enforced by the Pauli principle. The potential energy surface typically contained up to two minima, one spherical and one deformed. The analysis identified both first- and secondorder phase transitions for the PACM and the SACM, while in the latter case a critical line was also found.

The results were illustrated with numerical studies on the ${ }^{16} \mathrm{O}+\alpha$ and ${ }^{20} \mathrm{Ne}+\alpha$ systems, which correspond to two spherical clusters and to one spherical and one deformed cluster, respectively. The $S U(3)$ limit was found to be the most appropriate one in reproducing the data of the cluster systems. Clear phase transitions were identified in the $x$ parameter controlling the transition between the $S U(3)$ and $S O(4)$ limits. It was found that the PACM led to energy spectra that are rather different from the observed physical ones.

\section{Acknowledgments}

We gratefully acknowledge financial help from DGAPA, from the National Research Council of Mexico (CONACyT), OTKA (grant No. K72357), and from the MTA-CONACyT joint project. POH acknowledges very useful discussions with Octavio Castaños (ICN-UNAM), related to the differences in phase transitions in finite systems to the use of coherent states. The authors are also thankful to József Cseh for illuminating discussions on the subject.
[1] H. Yépez-Martínez, P. R. Fraser, P. O. Hess, and G. Lévai, Paper I.

[2] J. Cseh, Phys. Lett. B 281 (1992), 173.

[3] J. Cseh and G. Lévai, Ann. Phys. (N.Y.) 230 (1994), 165.

[4] F. Iachello, Phys. Rev. C 23 (1981), 2778.

[5] K. Wildermuth and Y. C. Tang, A Unified Theory of the Nucleus (Friedr. Vieweg \& Sohn Verlagsgesselschaft mbH, Braunschweig, 1977).

[6] P. O. Hess, G. Lévai and J. Cseh, Phys. Rev. C54 (1996), 2345.
[7] O. S. Roosmalen, Algebraic Description of Nuclear and Molecular Rotation-Vibration Spectra, (Ph.D. Thesis, Groningen, Netherland, 1982).

[8] O. S. Roosmalen and A. E. L. Dieperink, Ann. Phys. (N.Y.) 139 (1982), 198.

[9] A. Leviatan and M. W. Kirson, Ann. Phys. (N.Y.) 188 (1988), 142

[10] W. Greiner, L. Neise and H. Stöcker, Thermodynamcis and Statistical Mechanics, (Springer, Heidelberg, 1995).

[11] E. López-Moreno and O. Castaños, Phys. Rev. C 54 
(1996), 2374

[12] O. Castaños, R. López-Peña, J. G. Hirsch and E. LópezMoreno, Phys. Rev. B 74 (2006), 104118.

[13] R. Gilmore, Catastrophe Theory for Scientists and Engineers (Wiley, New York, 1981).

[14] H. Yépez-Martínez, J. Cseh and P. O. Hess, Phys. Rev. C 74 (2006), 024319.

[15] L. Parra Rodríguez, Master Thesis, UNAM, 2011.

[16] P. R. Fraser, H. Yépez-Martínez, P. O. Hess and L. ParraRodríguez, J. Phys. Conf. Proc. (2011), accepted.
[17] Cejnar P and Iachello F, 2007 J. Phys. A 40581

[18] Y. Zhang, Z.-F. Hou, H. Chen, H. Wei and Y.-X. Liu, Phys. Rev. C 78 (2008), 024314.

[19] A. Frank, P. Van Isacker, Symmetry Methods in Molecules and Nuclei, (SyG editores, México D.F., 2005).

[20] G. Rosensteel and J. P. Draayer, Nucl. Phys. A 436 (1985), 445.

[21] R. Bijker and F. Iachello, Ann. Phys. 298 (2002), 334. 